\section{Heme Oxygenase-1 Deficiency}

The oxidation of heme to biliverdin is facilitated by a stressinduced enzyme, heme oxygenase-1. This enzyme has antioxidant properties and plays an important role against inflammation. First case of heme oxygenase-1 deficiency was reported in 1999 [1]. The key features of heme oxygenase-1 deficiency, a recently described disorder, are hemolysis, generalized inflammation, bleeding diathesis, nephropathy and asplenia [1,2].

An 8-month old child presented with fever for 1-month. He was first of nonconcordant twins, and the sibling was healthy. On examination, he had dysmorphic facies, frontal bossing, depressed nasal bridge and large ears. General examination revealed pallor, and on systemic examination he had hepatomegaly. Investigation revealed increased inflammatory markers [raised CRP ( $84 \mathrm{mg} / \mathrm{L})$ and ferritin $(3503 \mathrm{ng} / \mathrm{mL})$ ], features of hemolysis [raised LDH (5253 U/L), SGOT (210 units/ L) and SGPT (114 units/L)], anemia and thrombocytopenia. He was worked up for bicytopenia (EBV, Parvo virus and CMV work up was negative). Other infections like tuberculosis and HIV were also ruled out. Bone marrow examination revealed few hemophagocytes. His initial USG abdomen revealed a normal spleen but on repeat $\mathrm{CT}$ abdomen after 6 months, spleen was not seen and only a small focal nodular calcified area within splenic fossa was seen. In view of the unclear primary diagnosis, hemophagocytes on marrow, increased liver enzymes and bicytopenia, he was started on oral steroids, pending further investigations. He improved, fever disappeared and liver enzymes returned to normal, but he needed transfusion once in 2-3 months. However, on tapering steroids, fever reappeared and he became pale again. He was readmitted for investigations. USG showed absent spleen. Hemoglobin electrophoresis showed sickle cell trait.

In view of features suggestive of autoinflammatory disorder, transfusion dependent anemia, and auto-splenectomy, clinical exome sequencing was done, which revealed homozygous nonsense variation in exon 3 of $H M O X 1$ gene (OMIM*141250) which causes human heme hemoxygenase-1 deficiency, confirmed by Sanger sequencing. Both parents were asymptomatic heterozygous carriers of the pathogenic variation detected in our patient.

When comparing our case with previous cases published in literature, we found our child had delayed development, growth retardation and dysmorphic features as reported earlier. $\mathrm{He}$ presented with fever but did not have lymphadenopathy or rash as seen in earlier cases. Also, he did not have asplenia from the beginning but had autosplenectomy during the course of treatment. Laboratory features similar in our case to the previous cases were, features of hemolysis (raised LDH and SGOT), raised inflammatory markers (raised CRP, ferritin). Our case is different from previously reported cases as he did not have coagulation abnormalities, features of nephritis or abnormal lipid profile [1-3]. Subsequently our patient developed acute arterial stroke confirmed on MRI and later was transfusion dependent. He succumbed to his illness at the age of $3 \frac{1}{2}$ years at a peripheral hospital.

Human heme oxygenase- 1 deficiency is a disease which is known to be associated with impaired stress hematopoiesis. This results in marked red blood cell fragmentation, intravascular hemolysis, coagulation abnormalities and endothelial damage. This leads to deposits in the kidney and liver. Clinical features include persistent hemolytic anemia, asplenia, nephritis, generalized erythematous rash, growth retardation and hepatomegaly. There is one case report of successful HLA matched stem cell transplantation in the literature.

Though a rare disorder, if a patient presents with features of hemolysis, generalized inflammation, bleeding diathesis, nephropathy and asplenia, diagnosis of human heme oxygenase-1 deficiency should be considered. All the features may not be present as our patient did not have coagulation abnormalities, features of nephritis or abnormal lipid profile. From our case, it is evident that the child had autosplenectomy, whereas literature suggests congenital asplenia.

SoumYa ReNJI, ${ }^{{ }^{*}}$ NitIN SHAH ${ }^{1}$ AND
MANISHA MADKAIKAR ${ }^{2}$
${ }^{1}$ Department of Pediatrics,
PD Hinduja National Hospital and
Medical Research Centre; and
${ }^{2}$ ICMR-National Institute of Immunohematology;
Mumbai, Maharashtra, India.
${ }^{*}$ soumya1577@gmail.com

\section{REFERENCES}

1. Kawashima A, Oda Y, Yachie A, et al. Heme oxygenase-1 deficiency: The first autopsy case. Hum Pathol. 2002;33: 125-30.

2. Radhakrishnan N, Yadav SP, Sachdeva A, et al. Human heme oxygenase-1 deficiency presenting with hemolysis, nephritis, and asplenia. J Pediatr Hematol Oncol. 2011; 33:74-8.

3. Jarmi T, Agarwal A. Heme oxygenase and renal disease. Curr Hyperten Rep. 2009;11:56-62.

4. Yadav SP, Thakkar D, Kohli S, Nivargi S, Rastogi N. Human heme-oxygenase-1 deficiency treated successfully by matched sibling donor allogeneic stem cell transplant. Biol Blood Marrow Transplant. 2018;24:S443. 